\title{
Thermal and back-action noises in dual-sphere gravitational-wave detectors
}

\author{
T. Briant, ${ }^{1}$ M. Cerdonio, ${ }^{2}$ L. Conti, ${ }^{2}$ A. Heidmann, ${ }^{1}$ A. Lobo,${ }^{3}$ and M. Pinard ${ }^{1}$ \\ ${ }^{1}$ Laboratoire Kastler Brossel, CNRS, Ecole Normale Supérieure and Université P. et M. Curie, Case 74, 4 place Jussieu, \\ F75252 Paris Cedex 05, France \\ ${ }^{2}$ INFN Padova Section and Department of Physics, University of Padova, via Marzolo 8, I-35100 Padova, Italy \\ ${ }^{3}$ Departament de Fisica Fonamental, Universitat de Barcelona, Diagonal 647, E-08028 Barcelona, Spain
}

(Received 14 January 2003; published 22 May 2003)

\begin{abstract}
We study the sensitivity limits of a broadband gravitational-wave detector based on dual resonators such as nested spheres. We determine both the thermal and back-action noises when the resonators' displacements are read out with an optomechanical sensor. We analyze the contributions of all mechanical modes, using a new method to deal with the force-displacement transfer functions in the intermediate frequency domain between the two gravitational-wave sensitive modes associated with each resonator. This method gives an accurate estimate of the mechanical response, together with an evaluation of the estimate error. We show that very high sensitivities can be reached on a wide frequency band for realistic parameters in the case of a dual-sphere detector.
\end{abstract}

DOI: 10.1103/PhysRevD.67.102005

PACS number(s): 04.80.Nn, 42.50.Lc, 95.55.Ym

\section{INTRODUCTION}

Dual systems, in the form of nested spheres $[1,2]$ and nested cylinders [3], have been recently proposed as wideband gravitational-wave detectors, of spectral sensitivity complementary in frequency to advanced interferometric detectors. Dual spheres are based on two spherical masses nested together: the inner mass is a full sphere while the other one is a hollow sphere. The radii match so that only a small gap separates the two bodies.

The fundamental modes of both spheres are quadrupole modes sensitive to gravitational waves. By a proper choice of the mechanical and geometrical characteristics of the two spheres, the fundamental mode of the inner sphere occurs at a frequency 2 or 3 times larger than the fundamental mode of the hollow one, without any sensitive modes in the intermediate frequency domain between these two fundamental modes. This frequency domain is of particular interest as the spheres displacements caused by a gravitational wave are out of phase by $\pi$ radians, thus leading to a measurable variation of the gap between the spheres.

To get a wideband detector, very sensitive and nonresonant displacement sensors are needed. An efficient technique consists in using optomechanical sensors based on a highfinesse Fabry-Perot cavity [4-6]. One mirror of the cavity is coated on the inner side of the hollow sphere, whereas the other mirror is coated on the solid sphere. The measurement of the phase of the field reflected by such a linear cavity thus provides information on the gap variation between the two spheres, at the radial position of the sensor. One can choose a strategy to set the number of optomechanical sensors and their location in such a way to reconstruct the sphere motion $[7,8]$.

A key point for broadband operation is the sensitivity of the optomechanical sensor which actually depends on its coupling with the mechanical modes of the system. Since the frequencies of interest are between two mechanical resonances, dual systems are conceptually different from bars and interferometers. With bars one has to deal with a single resonator, driven by the gravitational wave at its fundamental frequency. The treatment of the bar excitation by the gravitational wave, by the thermal noise in the bar and in the transducer, and by the back-action noise induced by the sensor is relatively straightforward, including the understanding of the standard quantum limit. One gets a prediction of the narrow-band spectral sensitivity as a function of relevant parameters, dealing basically with few isolated modes [9]. For the thermal noise, correlations may arise between the two modes originating from the tight coupling between the bar and the resonant transducer, when their mechanical quality factors are very different $[6,10]$, but the noise spectral behavior does not suffer dramatic changes.

In the case of interferometers the gravitational wave drives a set of masses, which can be considered free above the pendulum resonant frequencies of the suspensions. Again, the sources of noise can be spelled out, down to the quantum limit, and the wideband spectral sensitivity can be predicted as a function of relevant parameters [11].

Dual systems are conceptually different in that one has to deal neither at resonance as with bars, nor far from resonant modes as with interferometers, but rather in between resonant modes. The difficulty is then to write a mechanical transfer function for the system valid in this unusual frequency range.

In this paper we determine the limits induced both by the thermal noise of the spheres and by the quantum fluctuations of light, including the measurement noise and the backaction effects of light on the dual system. We show in the case of a single sensor that the limit of sensitivity can be expressed in terms of mechanical transfer functions characterizing the optomechanical coupling of light with the two spheres.

We illustrate this behavior in the case where only the excitation of fundamental modes of each sphere is taken into account, showing a new effect of back-action noise cancellation in the frequency domain between the two modes. This cancellation results from a destructive interference between radiation pressure effects on both spheres and leads to an 


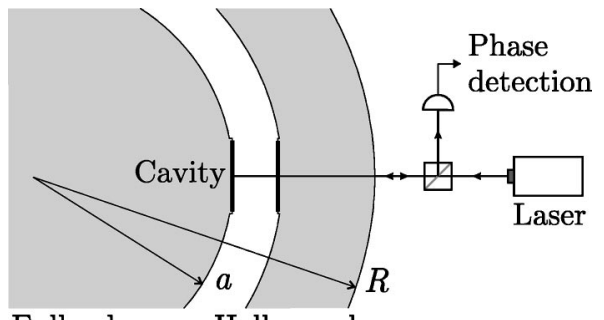

Full sphere Hollow sphere

FIG. 1. Scheme of the system studied in the paper. The small gap between the two spheres of the gravitational-wave detector (inner radius $a$, external radius $R$ ) is measured by an optomechanical sensor made of a high-finesse single-ended cavity. The phase of the reflected field is sensitive to the differential displacement of the mirrors.

increased sensitivity in between the two resonances.

We then develop a simple approach to take into account all other mechanical modes of the spheres. This method allows one to estimate the mechanical transfer functions in the intermediate frequency range between the resonances and gives an upper bound for the estimate error. As a result we determine the sensitivity of a dual-sphere detector and show that a spectral strain sensitivity better than $10^{-22} \mathrm{~Hz}^{-1 / 2}$ can be obtained over a wide frequency range from 1 to $3.5 \mathrm{kHz}$.

In Sec. II we present the basic principles of the gravitational-wave detection by a dual system with a transducer based on an optomechanical sensor. Section III is devoted to the determination of noises, illustrated in the case of a dual system with only two mechanical resonances. In Sec. IV we derive the mechanical transfer functions, taking into account all mechanical modes. Results for a beryllium dualsphere detector are presented in Sec. V.

\section{DUAL DETECTOR WITH AN OPTOMECHANICAL SENSOR}

The scheme of the dual-sphere detector is shown in Fig. 1. Although the main results of the paper are valid for any geometry of the dual system, we will consider the case of two nested spheres with an inner radius $a$ and an external radius $R$. The gap between the two bodies is taken to be small as compared to these radii. Typical dimensions are of the order of the meter for $a$ and $R$, and of a few centimeters for the gap.

The optomechanical sensor is based on a high-finesse single-ended cavity, resonant with the incident laser beam. The phase of the field reflected by the cavity is measured and compared to the phase reflected by a reference cavity in order to eliminate the effects of the laser frequency-noise [6].

As shown in $[12,13]$ the motion of one sphere (the full or hollow one) induces a global phase shift on the light proportional to the displacement $\hat{u}$ of the mirror surface averaged over the beam profile,

$$
\hat{u}(t)=\left\langle u_{\perp}(t), v_{0}^{2}\right\rangle,
$$

where the brackets stand for the overlap integral on the mirror surface,

$$
\langle f, g\rangle=\int d^{2} r f(r) g(r)
$$

$u_{\perp}(r, t)$ is the normal component of the displacement at point $r$ and time $t$, and $v_{0}^{2}(r)$ is the transverse Gaussian structure of the light intensity on the mirror,

$$
v_{0}^{2}(r)=\frac{2}{\pi w_{0}^{2}} e^{-2 r^{2} / w_{0}^{2}}
$$

where $w_{0}$ is the beam-spot size, identical for the two mirrors in the case of a symmetric cavity.

The optomechanical sensor then reads out the differential displacement $\hat{u}_{o}(t)-\hat{u}_{i}(t)$ between the internal surface of the outer sphere (subscript $o$ ) and the surface of the inner sphere (subscript $i$ ).

A gravitational wave induces a displacement which can be written as a sum over all quadrupole modes $\{n, 2\}$ of the spheres $[14,15]$. As long as the beam-spot size is small compared to the sphere radii, the spatial overlap between these modes and the beam profile is independent of $n$ and of the spot size. The radial displacement of each sphere, for a gravitational wave of amplitude $\widetilde{h}[\Omega]$ at frequency $\Omega$, is then equal to [16]

$$
\hat{u}^{g w}[\Omega]=-\frac{1}{2} \sum_{n=1}^{\infty} b_{n} A_{n 2}(a) \Omega^{2} L_{n 2}[\Omega] \widetilde{h}[\Omega],
$$

where $b_{n}$ are the coefficients in the orthogonal expansion of the response function of the sphere, $A_{n 2}(a)$ are the radial functions at the surface position $a$ (assumed to be the same for both surfaces since the gap is small), and $L_{n 2}[\Omega]$ are the frequency dependences of the modes $[14,15]$. They correspond to harmonic oscillators with resonance frequencies $\Omega_{n 2}$ and loss angle $\phi$, assumed to be the same for all modes and independent of frequency:

$$
L_{n 2}[\Omega]=\frac{1}{\Omega_{n 2}^{2}-\Omega^{2}-i \Omega_{n 2}^{2} \phi} .
$$

To illustrate the relevant features of the detector response we consider a dual-sphere made of beryllium with an inner radius $a=1.2 \mathrm{~m}$ and an outer radius $R=2 \mathrm{~m}$. For these parameters the fundamental frequencies are equal to $1161 \mathrm{~Hz}$ for the outer sphere and to $3075 \mathrm{~Hz}$ for the inner one. Products $b_{1} A_{12}(a)$ are equal to 1 for the hollow sphere and to 0.6 for the solid one. As a consequence, the displacements $\hat{u}_{i}^{g w}$ and $\hat{u}_{o}^{g w}$ are in phase for frequencies outside the two resonances and are out of phase by $\pi$ radians in the intermediate frequency domain between the two gravitational-wave sensitive modes. For these frequencies, effects of both modes are added to each other in the measured difference $\hat{u}_{o}^{g w}-\hat{u}_{i}^{g w}$.

Figure 2 shows the displacements induced by a gravitational wave of amplitude $\widetilde{h}=10^{-22} \mathrm{~Hz}^{-1 / 2}$, for each fundamental mode (curves $a$ and $b$ ). Curve $c$ is the global response due to the two fundamental modes. The response 


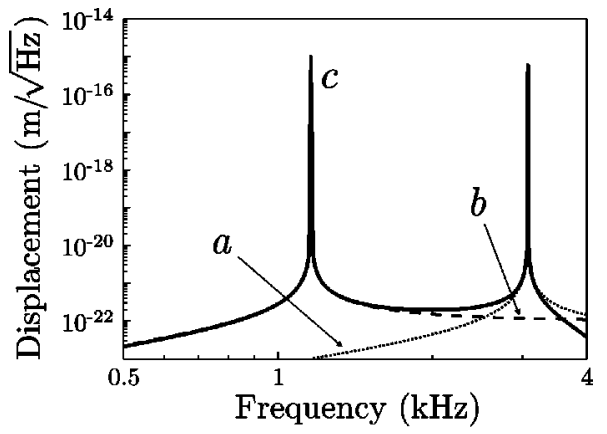

FIG. 2. Displacement $\left|\hat{u}_{o}^{g w}-\hat{u}_{i}^{g w}\right|$ as a function of frequency for a gravitational wave of amplitude $\widetilde{h}=10^{-22} \mathrm{~Hz}^{-1 / 2}$. Curves correspond to the response of the fundamental mode of the inner sphere $(a)$, of the fundamental mode of the outer sphere $(b)$, and of the two modes together $(c)$.

enhancement in the intermediate frequency domain is clearly visible, resulting in a flat response between the two fundamental resonances.

We have also determined the effect of higher modes by a numerical computation of their mechanical characteristics [14]. Taking into account modes with resonance frequencies up to $30 \mathrm{kHz}$ for the solid sphere and up to $16 \mathrm{kHz}$ for the hollow one, we obtain no significant change for the response in the frequency band between the two fundamental modes. This is related to the frequency dependence $\Omega^{2} / \Omega_{n 2}^{2}$ in the contribution of higher modes $\left(\Omega_{n 2} \gg \Omega\right)$ in the sum (4).

\section{THERMAL AND QUANTUM NOISES}

We now determine the classical and quantum noises in the measurement made by the optomechanical sensor. The phase of the field reflected by the cavity reproduces the cavity length variations. For a lossless cavity at resonance, the fluctuations $\delta q^{\text {out }}[\Omega]$ at frequency $\Omega$ for the phase quadrature of the reflected field is given by [12]

$$
\delta q^{\text {out }}[\Omega]=\delta q^{i n}[\Omega]+16 \bar{\alpha}^{i n} \mathcal{F} \frac{\hat{u}_{o}[\Omega]-\hat{u}_{i}[\Omega]}{\lambda},
$$

where $\delta q^{i n}$ are the phase quadrature fluctuations of the incident field, $\mathcal{F}$ the cavity finesse, $\lambda$ the optical wavelength, and $\bar{\alpha}^{i n}$ the mean incident field, related to the incident power $P^{i n}$ and to the wave vector $k=2 \pi / \lambda$ by

$$
P^{i n}=\hbar c k\left|\bar{\alpha}^{i n}\right|^{2}
$$

We have neglected in Eq. (6) the low-pass filtering due to the cavity bandwidth. Even for a cavity finesse $\mathcal{F}=10^{6}$ and a gap between the two spheres of $1 \mathrm{~cm}$, the cavity bandwidth is larger than $7.5 \mathrm{kHz}$ and has no significant effect at frequencies of interest. We also assume that the incident beam is in a coherent state, so that phase quadrature $\delta q^{i n}$ and intensity quadrature $\delta p^{i n}$ are uncorrelated with noise spectra given by [12]

$$
S_{p p}^{i n}[\Omega]=S_{q q}^{i n}[\Omega]=1 .
$$

The phase of the reflected beam provides a measurement of the displacements induced by a gravitational wave, with additional noises related to the incident phase fluctuations and to displacement noises [Eq. (6)]. The main sources of displacement noise are the thermal fluctuations of spheres and the back action of the measurement process due to the radiation pressure exerted by the intracavity field on both mirrors. All these noises are uncorrelated and the spectral strain sensitivity is given by equating the contribution of the gravitational-wave signal with the noise contributions,

$$
S_{h h}[\Omega]=\frac{S_{u u}^{\text {shot }}[\Omega]+S_{u u}^{t h}[\Omega]+S_{u u}^{b a}[\Omega]}{\left|\hat{u}_{o}^{g w}[\Omega]-\hat{u}_{i}^{g w}[\Omega]\right|^{2} /|\widetilde{h}[\Omega]|^{2}},
$$

where $S_{u u}^{\text {shot }}, S_{u u}^{t h}$, and $S_{u u}^{b a}$ are the displacement noise spectra due to incident phase fluctuations, thermal noise, and back action, respectively.

We now determine these three noise spectra. The shot noise $S_{u u}^{\text {shot }}$ is given by the equivalent displacement noise corresponding in the measurement to the phase quadrature fluctuations $\delta q^{i n}$. From Eqs. (6) and (8) one gets

$$
S_{u u}^{\text {shot }}[\Omega]=\frac{\lambda^{2}}{256 \mathcal{F}^{2}\left|\bar{\alpha}^{i n}\right|^{2}} .
$$

Thermal fluctuations of the two spheres are uncorrelated and their contributions are added to each other in the thermal noise spectrum $S_{u u}^{t h}$. For each sphere, the displacement can be related to a Langevin force $F^{t h}$ describing the coupling with a thermal bath [17],

$$
\hat{u}^{t h}[\Omega]=\chi[\Omega] F^{t h}[\Omega],
$$

where $\chi[\Omega]$ is a mechanical susceptibility characterizing the response of the sphere to an external force. Since it relies on the displacement $\hat{u}$ averaged over the beam-spot size, it depends on the spatial overlap with the light. Using a modal expansion for the displacements of the sphere, $\chi[\Omega]$ is given by $[12,13]$

$$
\chi[\Omega]=\sum_{n=1}^{\infty}\left\langle u_{n}, v_{0}^{2}\right\rangle^{2} \chi_{n}[\Omega],
$$

where $\left\langle u_{n}, v_{0}^{2}\right\rangle$ is the spatial overlap between the mechanical mode $u_{n}(r)$ and the beam profile [Eq. (3)]. $\chi_{n}[\Omega]$ is the mechanical susceptibility associated with mode $n$,

$$
\chi_{n}[\Omega]=\frac{1}{M_{n}\left(\Omega_{n}^{2}-\Omega^{2}-i \Omega_{n}^{2} \phi\right)},
$$

where $\Omega_{n}$ is the resonance frequency of the mode and $M_{n}$ its effective mass related to the total mass $M$ and to the volume $V$ of the sphere by

$$
M_{n}=\frac{M}{V} \int_{V} d^{3} r\left|u_{n}(r)\right|^{2}
$$


The noise spectrum of the Langevin force $F^{\text {th }}$ is related to the mechanical susceptibility through the fluctuationsdissipation theorem [17],

$$
S_{F F}^{t h}[\Omega]=-\frac{2 k_{B} T}{\Omega} \operatorname{Im}\left(\chi[\Omega]^{-1}\right),
$$

where $k_{B}$ is the Boltzmann constant and $T$ the temperature. From Eqs. (11) and (15) one then gets the noise spectrum for the thermal fluctuations of the differential displacement $\hat{u}_{o}$ $-\hat{u}_{i}$ measured by the optomechanical sensor

$$
S_{u u}^{t h}[\Omega]=\frac{2 k_{B} T}{\Omega} \operatorname{Im}\left(\chi_{o}[\Omega]+\chi_{i}[\Omega]\right),
$$

where $\chi_{o}$ and $\chi_{i}$ are the mechanical susceptibilities of the outer and inner spheres, respectively.

Back-action effects are related to the radiation pressure forces exerted by the intracavity field on both mirrors. Since the radiation pressure has the same spatial profile as the intracavity intensity, it can be shown [13] that the displacement $\hat{u}^{b a}$ of each sphere is related to the force through the same susceptibility as the thermal noise [Eq. (12)],

$$
\hat{u}^{b a}[\Omega]=\chi[\Omega] F^{b a}[\Omega] .
$$

The radiation pressure force is equal to

$$
F^{b a}[\Omega]=2 \hbar k I[\Omega],
$$

where $I$ is the intracavity intensity normalized as a number of photons per second. Intensity fluctuations can be related to the amplitude quadrature of the incident field [12] and one gets the noise spectrum of the radiation pressure,

$$
S_{F F}^{b a}[\Omega]=\frac{16 \hbar^{2} k^{2}}{\pi^{2}} \mathcal{F}^{2}\left|\bar{\alpha}^{i n}\right|^{2} .
$$

The radiation pressure forces exerted on the two mirrors are equal and opposite. The induced cavity-length variation is therefore related to the radiation pressure force through the sum of the susceptibilities of the outer and inner resonators,

$$
\hat{u}_{o}^{b a}[\Omega]-\hat{u}_{i}^{b a}[\Omega]=\left(\chi_{o}[\Omega]+\chi_{i}[\Omega]\right) F^{b a}[\Omega],
$$

and the resulting noise spectrum is given by,

$$
S_{u u}^{b a}[\Omega]=\left|\chi_{o}[\Omega]+\chi_{i}[\Omega]\right|^{2} S_{F F}^{b a}[\Omega] .
$$

We have thus determined the spectra of the three fundamental noises appearing in the measurement [Eqs. (10), (16), and (21)]. They only depend on a few parameters. The optical properties are accounted for via the cavity finesse $\mathcal{F}$ and the incident light intensity $\left|\bar{\alpha}^{i n}\right|^{2}$, with the usual behavior that the shot noise is decreased for a larger cavity finesse or a more intense light, whereas the back-action noise is increased [Eqs. (10) and (19)]. This behavior leads to the standard quantum limit $[18,19]$. The mechanical characteristics only depend on the sum $\chi_{o}+\chi_{i}$ of the susceptibilities of the two spheres.

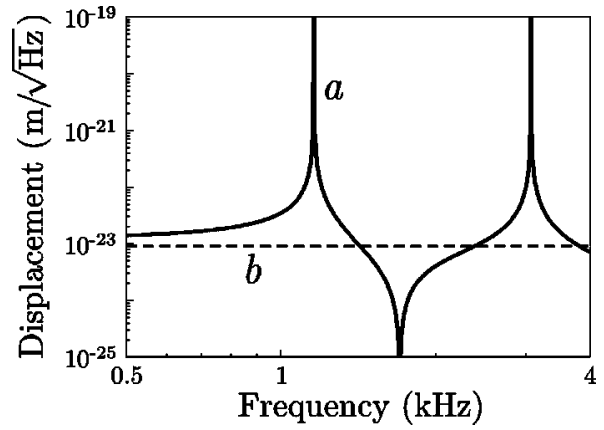

FIG. 3. Displacement noises as a function of frequency for back-action effect ( $\sqrt{S_{u u}^{b a}}$, curve $a$ ) and for shot-noise $\left(\sqrt{S_{u u}^{\text {shot }}}\right.$, curve $b$ ). Only the two fundamental modes of the spheres are taken into account.

To understand the relevant features of the quantum noises we consider a beryllium dual-sphere at zero temperature $\left(S_{u u}^{t h}=0\right)$, neglecting all mechanical modes except the fundamental modes of the two spheres. The sum in expression (12) of the mechanical susceptibilities is then limited to the first term $n=1$, their frequency dependence being the same as the response to a gravitational wave [compare Eqs. (5) and (13)]. In contrast to this response, radiation pressure forces exerted on the two mirrors are opposite and one gets a backaction noise cancellation in the intermediate frequency domain between the two fundamental resonance frequencies. The mechanical susceptibilities $\chi_{o}$ and $\chi_{i}$ actually have opposite signs in between the two resonances so that the effects of both spheres interfere destructively in the global backaction noise $S_{u u}^{b a}$ [Eq. (21)].

Figure 3 clearly shows this back-action cancellation, resulting in an important noise reduction on the whole frequency band between the two resonances (curve $a$ ). The optical parameter $\mathcal{F}^{2}\left|\bar{\alpha}^{i n}\right|^{2}$ is chosen in such a way that back-action and shot noises are equal at low frequency. The frequency for which exact cancellation occurs depends on the effective masses of the fundamental modes [Eqs. (13) and (14)] and can be tuned in the intermediate frequency domain by changing the mechanical characteristics of the spheres.

Since the contributions of both modes to the signal are added to each other whereas the back-action noises are subtracted, one can reach a very high sensitivity in the intermediate frequency domain, as shown in Fig. 4. Curve $b$ is obtained for the same optical parameter as Fig. 3 and exhibits a high sensitivity over a wide bandwidth. The behavior of the spectral strain sensitivity actually depends on the ratio between the shot and back-action noises. For a ten-times smaller optical parameter (curve $a$ ), shot noise is dominant and one recovers two peaks at the fundamental resonances frequencies. For a ten-times larger optical parameter (curve $c$ ), the sensitivity follows the back-action noise behavior and reaches an optimum value near the frequency for which back-action is cancelled out.

Curve $d$ in Fig. 4 shows the standard quantum limit which corresponds to the minimum reachable noise for a coherent incident light. It is obtained by adjusting at every frequency 


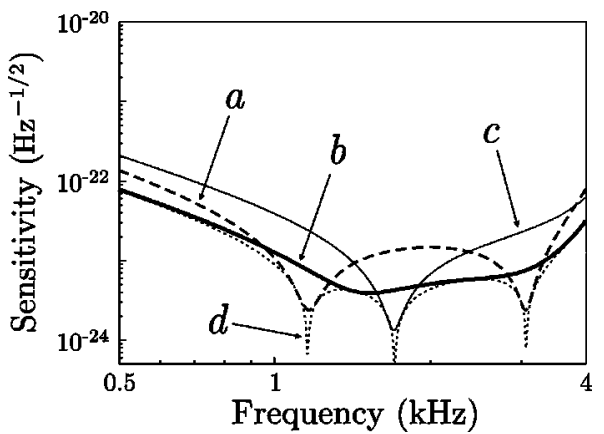

FIG. 4. Quantum-limited spectral strain sensitivity $\sqrt{S_{h h}}$ as a function of frequency for increasing incident intensities (curves $a$ to $c$ ). Curve $d$ is the standard quantum limit. Only the two fundamental modes of the spheres are taken into account.

the optical parameter $\mathcal{F}^{2}\left|\bar{\alpha}^{i n}\right|^{2}$ in such a way that shot and back-action noises are equal. From Eqs. (10), (19), and (21), the resulting minimum displacement noise is given by

$$
S_{u u}^{s q l}[\Omega]=\hbar\left|\chi_{o}[\Omega]+\chi_{i}[\Omega]\right| .
$$

As with other noises, it depends on the mechanical characteristics only via the sum of the susceptibilities of the two spheres.

\section{MECHANICAL SUSCEPTIBILITY}

To perform an accurate evaluation of the spectral strain sensitivity of the detector, we need to calculate the mechanical susceptibilities of the two spheres. For this purpose we can use two different approaches, but both are insufficient for our needs.

The first one is the normal modes expansion $[13,20,21]$ which can be used in principle in any case, if inhomogeneous losses are absent (it fails in the presence of inhomogeneous losses since correlations arise between modes [6,10,22]). This method consists of computing the sum in Eq. (12) over a finite number $N$ of modes,

$$
\chi^{(N)}[\Omega]=\sum_{n=1}^{N}\left\langle u_{n}, v_{0}^{2}\right\rangle^{2} \chi_{n}[\Omega] .
$$

This quantity of course converges towards the mechanical susceptibility for a large number $N$ of modes. It unfortunately converges very slowly, and the computation of the appropriate overlap integrals between the modes and light profiles, for higher and higher modes, is in practice too demanding.

The second approach applies in a direct manner the fluctuations-dissipation theorem to estimate the thermal noise, bypassing any normal mode expansion [23-25]. This global approach has been worked out in the limit of very low frequencies, so the results can be only used well below any resonance frequency of the system. It actually gives the static susceptibility in the case where the size of the sensor is small compared to the dimensions of the system. For the dual sphere, assuming the beam-spot size $w_{0}$ is small compared to the spheres radii, the sphere surface can be approximated by a half-infinite plane and one gets [26]

$$
\chi[\Omega=0]=\frac{1-\sigma^{2}}{\sqrt{\pi}(1-i \phi) E w_{0}},
$$

where $E$ and $\sigma$ are, respectively, the Young modulus and the Poisson coefficient of the sphere.

We want to calculate the susceptibilities for frequencies near or between resonance frequencies of the system so that we cannot apply this global approach. We instead consider a new method which simultaneously uses the modal and global approaches. We write the mechanical susceptibility in the form

$$
\chi[\Omega]=\chi[0]+\lim _{N \rightarrow \infty}\left(\chi^{(N)}[\Omega]-\chi^{(N)}[0]\right) .
$$

The first term is determined using the global approach [Eq. (24)] whereas the difference $\chi^{(N)}[\Omega]-\chi^{(N)}[0]$ is computed using the modal approach [Eq. (23)]. We perform the sum over a finite number $N$ of modes such that the resonance frequency $\Omega_{N}$ is much larger than the frequency $\Omega$ of interest.

The advantage of this approach is that the difference in Eq. (25) converges more quickly than each term taken separately. As a matter of fact, the difference is given by

$$
\chi^{(N)}[\Omega]-\chi^{(N)}[0]=\sum_{n=1}^{N}\left\langle u_{n}, v_{0}^{2}\right\rangle^{2}\left(\chi_{n}[\Omega]-\chi_{n}[0]\right) .
$$

From Eq. (13) the term in the sum for a mode $n$ such that $\Omega_{n} \gg \Omega$ can be bounded by

$$
\chi_{n}[\Omega]-\chi_{n}[0] \leqslant \chi_{n}[0] \frac{\Omega^{2}}{\Omega_{n}^{2}-\Omega^{2}}
$$

The last factor $\Omega^{2} /\left(\Omega_{n}^{2}-\Omega^{2}\right)$ ensures a rapid convergence of the difference $\chi^{(N)}[\Omega]-\chi^{(N)}[0]$ as compared to the one of $\chi^{(N)}[0]$.

It is furthermore possible to determine an upper bound for the error $\Delta \chi$ made by computing Eq. (26) up to a mode $N$. $\Delta \chi$ is given by

$$
\Delta \chi[\Omega]=\sum_{n=N+1}^{\infty}\left\langle u_{n}, v_{0}^{2}\right\rangle^{2}\left(\chi_{n}[\Omega]-\chi_{n}[0]\right) .
$$

Using Eq. (27) for modes $n$ such that $\Omega_{n} \geqslant \Omega_{N}$ one gets

$$
\Delta \chi[\Omega] \leqslant \chi[0] \frac{\Omega^{2}}{\Omega_{N}^{2}-\Omega^{2}} .
$$

This upper limit shows that the computation is accurate within $1 \%$ if the sum is extended up to modes with resonance frequencies ten times larger than the frequency $\Omega$ of interest.

The approach presented in this section can be used to determine the thermal and back-action noises when one motion sensor is used as read out in any dual-resonator system. It can also be extended to calculate the spatial correlations 


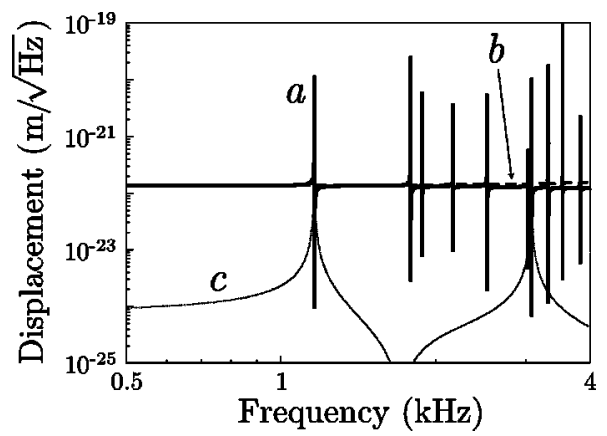

FIG. 5. Displacement noises as a function of frequency for back-action effect ( $\sqrt{S_{u u}^{b a}}$, curve $a$ ) and for shot-noise ( $\sqrt{S_{u u}^{\text {shot }}}$, curve $b$ ). Curve $c$ shows the contribution of the two fundamental modes to the back-action noise.

between modes. This would be needed to discuss the effects on thermal and back-action noises, when using a multitransducer read-out configuration.

\section{SENSITIVITY OF A DUAL SPHERE}

We apply the approach presented in the previous section to determine the mechanical susceptibilities $\chi_{o}$ and $\chi_{i}$ for a dual sphere. As previously we consider spheres made of beryllium with radii $a=1.2 \mathrm{~m}$ and $R=2 \mathrm{~m}$. To calculate the mechanical susceptibilities we take into account the contributions of 120 modes for the solid sphere (resonance frequencies up to $30 \mathrm{kHz}$ ) and of 80 modes for the hollow one (up to $16 \mathrm{kHz}$ ) [14]. Optical parameters are as follows: wavelength $\lambda=1 \mu$, beam-spot size on the mirrors $w_{0}$ $=1 \mathrm{~cm}$, cavity finesse $\mathcal{F}=10^{6}$, and incident power $P^{\text {in }}$ $=45 \mathrm{~mW}$.

An important issue is to reach such a large beam waist in an optical cavity with a length limited to a few centimeters. A possibility recently proposed in [27] is to use a folded Fabry-Perot cavity in which the light beam experiences several reflections on the two sphere surfaces before getting back to the input mirror. Such a cavity maintains the sensitivity of the measurement, but with a larger effective waist. Another possibility [28] is to use a convex-concave cavity, made of a convex mirror of radius $-R_{1}$ and a concave mirror of radius $R_{2}$, with $R_{2}>R_{1}$. The cavity is stable if the cavity length $L$ is larger than $R_{2}-R_{1}$. For values of $L$ and $R_{2}-R_{1}$, small as compared to $R_{1}$ and $R_{2}$, the beam-spot size $w_{0}$ in the cavity is given by

$$
w_{0}^{2}=\frac{\lambda R_{1}}{\pi} \sqrt{\frac{L}{L-R_{2}+R_{1}}} .
$$

One gets a spot size larger than $1 \mathrm{~cm}$ for radii $R_{1}$ $=4000 \mathrm{~cm}, \quad R_{2}=4004 \mathrm{~cm}$, and a cavity length $L$ $=4.04 \mathrm{~cm}$.

Figure 5 shows the shot and back-action noises obtained by a numerical calculation of Eq. (25). The chosen optical parameters are such that the back-action noise (curve $a$ ) and the shot noise (curve $b$ ) are equal at low frequency. Curve $c$ is the noise obtained when only the two fundamental modes of the spheres are considered for the same optical param-

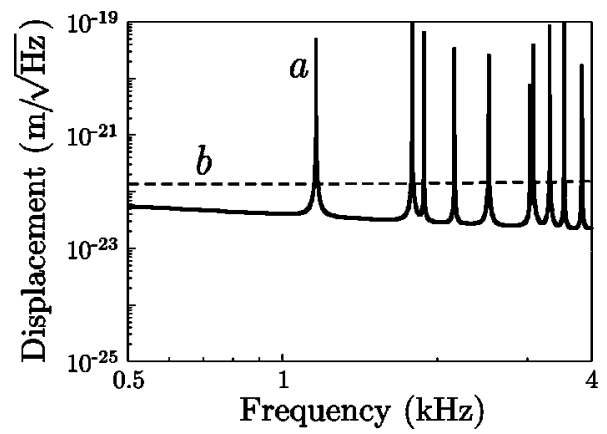

FIG. 6. Displacement noises as a function of frequency for thermal fluctuations $\left(\sqrt{S_{u u}^{t h}}\right.$, curve $a$ ) and for shot-noise $\left(\sqrt{S_{u u}^{\text {shot }}}\right.$, curve $b)$, in the same conditions as in Fig. 5 .

eters. Taking into account higher modes then drastically increases the back-action noise by two orders of magnitude. The resulting spectrum is almost flat in frequency and equal to the value obtained at zero frequency by considering only the static susceptibility $\chi[0][\mathrm{Eq} .(24)]$. The presence of resonant modes in the frequency band of interest leads to narrow peaks in the noise spectrum. They correspond to the two fundamental modes but also to other modes which are not sensitive to gravitational waves. Back-action cancellation is responsible for narrow dips after each resonance, at a frequency where the mechanical response of the resonant mode compensates the response of all other modes.

Figure 6 shows the thermal noise obtained for a quality factor equal to $10^{7}\left(\phi=10^{-7}\right)$ and a temperature $T$ $=0.1 \mathrm{~K}$. All modes of the dual sphere are thermally excited and responsible for the presence of peaks in the spectrum. For the chosen parameters the background thermal noise between two resonances will have no significant effect since it is smaller than the shot noise (curve $b$ in Fig. 6).

We have also considered the displacement noise due to thermodynamic and photothermal fluctuations. At low temperature, the adiabatic approximation [26] is no longer valid for beryllium since the heat diffusion length at frequencies of interest is much smaller than the beam-spot size. Thermoelastic noises are then almost independent on frequency and are only related to the thermal properties of beryllium (thermal expansion coefficient, thermal conductivity, and thermal capacity) [29]. Using the values of these parameters at $0.1 \mathrm{~K}[30,31]$ and assuming a mirror absorption less than $1 \mathrm{ppm}$, one finds that both the thermodynamic and photothermal noises are smaller than $10^{-23} \mathrm{~m} / \sqrt{\mathrm{Hz}}$. They have no significant contribution to the total noise.

Curve $a$ of Fig. 7 shows the resulting spectral strain sensitivity obtained from Eq. (9) by computing the response to a gravitational wave as explained in Sec. II. Except for narrow peaks due to the resonant behavior of thermal and backaction noises, one gets a quantum-limited sensitivity with optimums of the order of $2 \times 10^{-24} \mathrm{~Hz}^{-1 / 2}$ at the resonance frequencies of the two fundamental modes. The sensitivity is better than $10^{-22} \mathrm{~Hz}^{-1 / 2}$ over a wide frequency range, from 1 to $3.5 \mathrm{kHz}$.

The frequency dependence of the sensitivity is, however, very different from the one obtained in a two-modes analysis (Fig. 4). This clearly shows that two-modes or even few- 


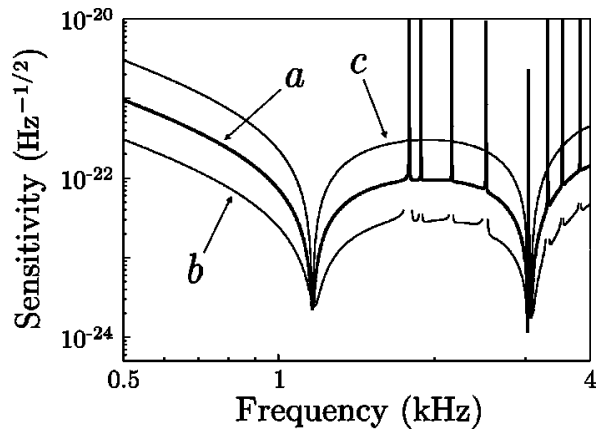

FIG. 7. Spectral strain sensitivity $\sqrt{S_{h h}}$ as a function of frequency for the same parameters as in Figs. 5 and 6 (curve $a$ ). Curves $b$ and $c$ correspond to a beam-spot size of 10 and $0.1 \mathrm{~cm}$, instead of $1 \mathrm{~cm}$. Narrow peaks of these curves have been removed for convenience.

modes analyses are not satisfactory to describe the mechanical behavior of the spheres. Taking into account all the modes leads to a larger mechanical response to radiation pressure fluctuations. As compared to Fig. 4 or to previous results [1] one gets sharper dips around the fundamental resonance frequencies and a less flat sensitivity between the two resonances.

Except near mechanical resonances, the response to quantum fluctuations is very similar to the static response given by the mechanical susceptibility $\chi[0]$. As shown in Eq. (24) a crucial parameter is then the beam-spot size $w_{0}$ on the mirrors. Curves $b$ and $c$ in Fig. 7 show the sensitivity for a beam-spot size of 10 and $0.1 \mathrm{~cm}$, respectively. In both cases the optical parameters are adjusted to stay at the standard quantum limit at low frequency. Curve $b$ corresponds to an incident power $P^{i n}=450 \mathrm{~mW}$ and curve $c$ to $4.5 \mathrm{~mW}$. It clearly appears that increasing the beam-spot size by a factor of 10 leads to wider dips at the fundamental resonance fre- quencies and to a gain of sensitivity between the resonances by a factor of $\sqrt{10}$, as expected from the expression of the standard quantum limit [Eqs. (22) and (24)].

\section{CONCLUSION}

We have determined the sensitivity of a dual-sphere detector taking into account the thermal, shot, and back-action noises. Neglecting all modes except the two fundamental ones, we obtained a very high sensitivity in the intermediate frequency domain between the two resonances, associated with a cancellation of back-action noise.

Using a new method mixing global and modal approaches, we have calculated the mechanical response of the dual-sphere. The features of the spectral strain sensitivity then look very different from the idealized two-modes model, resulting in sharper dips around the fundamental resonances and a less flat sensitivity between the two resonances.

An important parameter is the beam-spot size $w_{0}$ on the mirrors of the optomechanical sensor. Increasing $w_{0}$ reduces the influence of high-frequency modes, resulting in a better spectral strain sensitivity. A challenge is thus to design short Fabry-Perot cavities with large beam waists. One may envision to use either a convex-concave cavity or a folded FabryPerot cavity with large effective waists [27]. Another possibility is to use nonresonant capacitive and inductive transducers at their quantum limits, which intrinsically have a large sensor area. Finally one may try to implement a geometrically selective read out, as proposed for dual-cylinder [3] to reduce the influence of nonsensitive modes.

\section{ACKNOWLEDGMENT}

We gratefully thank F. Marin for stimulating discussions relative to the optics of the optomechanical sensor.
[1] M. Cerdonio et al., Phys. Rev. Lett. 87, 031101 (2001).

[2] L. Conti et al., Class. Quantum Grav. 19, 2013 (2002).

[3] M. Bonaldi et al., gr-qc/0302012.

[4] Y. Hadjar, P.F. Cohadon, C.G. Aminoff, M. Pinard, and A. Heidmann, Europhys. Lett. 47, 545 (1999).

[5] P.F. Cohadon, A. Heidmann, and M. Pinard, Phys. Rev. Lett. 83, 3174 (1999).

[6] L. Conti et al., J. Appl. Phys. 93, 3589 (2003).

[7] S.M. Merkowitz and W.W. Johnson, Phys. Rev. D 56, 7513 (1997).

[8] J.A. Lobo, Mon. Not. R. Astron. Soc. 316, 173 (2000).

[9] J.C. Price, Phys. Rev. D 36, 3555 (1987).

[10] E. Majorana and Y. Ogawa, Phys. Lett. A 233, 162 (1997).

[11] P. Hello, Progress in Optics XXXVIII, edited by E. Wolf (North Holland, Amsterdam, 1998), p. 85.

[12] A. Heidmann, Y. Hadjar, and M. Pinard, Appl. Phys. B: Lasers Opt. 64, 173 (1997).

[13] M. Pinard, Y. Hadjar, and A. Heidmann, Eur. Phys. J. D 7, 107 (1999).
[14] J.A. Lobo, Phys. Rev. D 52, 591 (1995).

[15] E. Coccia et al., Phys. Rev. D 57, 2051 (1998).

[16] We assume that the gravitational wave impinges with optimal direction and polarization with respect to the position of the sensor. A spherical detector with a proper multitransducer read out would be omnidirectional $[7,8]$.

[17] L. Landau and E. Lifshitz, Course of Theoretical Physics: Statistical Physics (Pergamon, New York, 1958), Chap. 12.

[18] C.M. Caves, Phys. Rev. D 23, 1693 (1981).

[19] V.B. Braginsky and F.Ya. Khalili, Quantum Measurement (Cambridge University Press, Cambridge, England, 1992).

[20] F. Bondu and J.Y. Vinet, Phys. Lett. A 198, 74 (1995).

[21] A. Gillespie and F. Raab, Phys. Rev. D 52, 577 (1995).

[22] K. Yamamoto et al., Phys. Lett. A 280, 289 (2001).

[23] Y. Levin, Phys. Rev. D 57, 659 (1998).

[24] F. Bondu, P. Hello, and J.Y. Vinet, Phys. Lett. A 246, 227 (1998).

[25] Y.T. Liu and K.S. Thorne, Phys. Rev. D 62, 122002 (2000).

[26] V.B. Braginsky, M.L. Gorodetsky, and S.P. Vyatchanin, Phys. Lett. A 264, 1 (1999). 
[27] F. Marin, L. Conti, and M. De Rosa, Phys. Lett. A 309, 15 (2003).

[28] F. Marin (private communication).

[29] M. Cerdonio, L. Conti, A. Heidmann, and M. Pinard, Phys.
Rev. D 63, 082003 (2001).

[30] American Institute of Physics Handbook, 3rd ed., edited by D.E. Gray (McGraw-Hill, New York, 1972).

[31] G. Ahlers, Phys. Rev. 145, 419 (1966). 